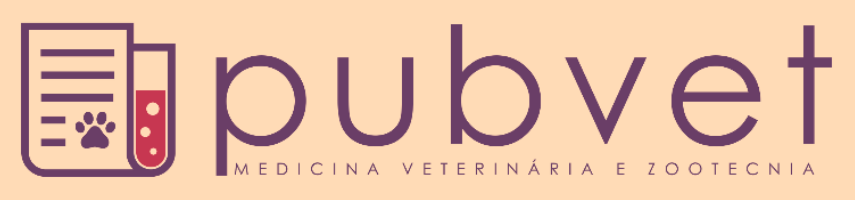

https://doi.org/10.31533/pubvet.v12n10a199.1-7

\title{
Análises microbiológicas de carne bovina moída comercializada em supermercados em Macapá, Amapá
}

\author{
Antonio Carlos Souza da Silva Júnior ${ }^{*} \bullet$, Jaqueline Freitas do Nascimento ${ }^{2} \bullet$, Ediluci do \\ Socorro Leôncio Tostes ${ }^{3}$, Anne do Socorro Santos da Silva ${ }^{4}$
}

${ }^{I}$ Pesquisador do Instituto de Pesquisas Científicas e Tecnológicas do Estado do Amapá-IEPA, Núcleo de Ciência e Tecnologia de Alimentos. Macapá-AP Brasil. ${ }^{2}$ Acadêmica de Farmácia, Instituto Macapaense de Ensino Superior-IMMES. Macapá-AP Brasil.

${ }^{3}$ Pesquisadora do Instituto de Pesquisas Científicas e Tecnológicas do Estado do Amapá-IEPA, Núcleo de Ciência e Tecnologia de Alimentos. Macapá-AP Brasil. ${ }^{4}$ Pesquisadora do Instituto de Pesquisas Científicas e Tecnológicas do Estado do Amapá-IEPA, Núcleo de Ciência e Tecnologia de Alimentos. Macapá-AP Brasil. *Autor para correspondência, E-mail: jr_bio2005@yahoo.com.br

RESUMO. Por ser um alimento rico em nutrientes (gorduras, proteínas, minerais e vitaminas) e de fácil acesso, a carne bovina moída é consumida em todo o território brasileiro. Todavia, durante o processo é um produto bastante manipulado que pode levar a alterações microbianas. Desta forma, o objetivo deste trabalho foi analisar as características microbiológicas de carne bovina moída comercializada em supermercados da cidade de Macapá, Amapá. Foram realizadas três coletas de amostras de carne bovina moída, em porções de $500 \mathrm{~g}$ de maneira aleatória direto dos balcões refrigerados em dez supermercados localizados em diferentes pontos da cidade de Macapá durante os meses de fevereiro a julho de 2018, totalizando 30 amostras. No momento da coleta, com o auxílio de termômetro infravermelho foi medida a temperatura. As amostras foram encaminhas para o Núcleo de Ciência e Tecnologia de Alimentos do Instituto de Pesquisas Científicas e Tecnológicas do Estado do Amapá-IEPA. As análises microbiológicas foram a presença/ausência de Salmonella spp. em $25 \mathrm{~g}$ de amostra e Contagem padrão de Staphylococcus coagulase positiva (UFC/g). Quanto a temperatura de exposição foi possível constatar que $8(88,9 \%)$ das amostras avaliadas estavam com temperaturas acima de $4^{\circ} \mathrm{C}$, não atendendo a legislação vigente. Quanto as análises microbiológicas realizadas neste estudo constataram-se ausência em todas as amostras para Salmonella spp.; porém, resultados positivos em $100 \%$ dos estabelecimentos avaliados para Staphylococcus coagulase positiva, onde a amplitude registrada foi de $2,3 \times 10^{3}$ a $2 \times 10^{5} \mathrm{UFC} / \mathrm{g}$. Os supermercados mesmo utilizando processos mecanizados durante as etapas de processamento e passando por um padrão de controle considerado mais exigente do que outros estabelecimentos, ainda foi possível contatar um número expressivo de contaminação por Staphylococcus, que mesmo que a legislação não preveja este microrganismo, ele ainda é considerado um dos agentes patogênicos mais envolvidos com surtos e casos esporádicos de intoxicações alimentares.

Palavras chave: carne processada, Salmonella, Staphylococcus

\section{Microbiological analysis of ground beef commercialized in supermarkets in Macapá, Amapá}

ABSTRACT. Because the meat is a food rich in nutrients (fat, protein, minerals and vitamins, and easily accessible, ground beef is widely consumed throughout Brazil. However, during the production process it is a highly manipulated product that can lead to microbial contamination. In this way, the objective of this work was to analyze the microbiological characteristics of ground beef commercialized in supermarkets in the city 
of Macapá, Amapá. Three samples of ground beef were taken in portions of $500 \mathrm{~g}$ randomly direct from the refrigerated counters in ten supermarkets located in different points of the city of Macapá during from February to July of 2018, totaling 30 samples. At the time of the collection, the infrared thermometer was used to measure the exposure temperature. The samples were sent to the Food Science and Technology Center of the Institute of Scientific and Technological Research of the State of Amapá-IEPA. The microbiological analyzes were the presence / absence of Salmonella spp. in $25 \mathrm{~g}$ of sample and Standard Coagulase-positive Staphylococcus Count (CFU / g). Regarding the temperature of exposure, it was possible to verify that $8(88.89 \%)$ of the samples evaluated had temperatures above $4{ }^{\circ} \mathrm{C}$, not complying with current legislation. The microbiological analyzes performed in this study showed absence in all samples for Salmonella spp., but positive results in $100 \%$ of the establishments evaluated for Staphylococcus coagulase positive, where the amplitude was $2.3 \times 103$ at $2 \times 105 \mathrm{CFU} / \mathrm{g}$. Supermarkets even using mechanized processes during the production stages and passing through a standard of control considered more demanding than other establishments, it was still possible to contact an expressive number of Staphylococcus contamination, that even if the legislation does not provide this microorganism, it is still considered one of the pathogens most involved with outbreaks and sporadic cases of food poisoning.

Keywords: Processed meat, Salmonella, Staphylococcus

\section{Análisis microbiológicos de la carne picada vendida en supermercados en Macapá, Amapá}

RESUMEN. Por ser un alimento rico en nutrientes (vitaminas, minerales y proteínas) y de fácil acceso, la carne bovina molida es ampliamente consumida en todo el territorio brasileño. Sin embargo, durante el proceso productivo es un producto bastante manipulado que puede llevar a cambios microbianos. De esta forma, el objetivo de este trabajo fue analizar las características microbiológicas de carne bovina molida comercializada en supermercados de la ciudad de Macapá-Amapá. Se realizaron tres colectas de muestras de carne bovina molida, en porciones de $500 \mathrm{~g}$ de forma aleatoria directa de los mostradores refrigerados en diez supermercados ubicados en diferentes puntos de la ciudad de Macapá durante los meses de febrero a julio de 2018, totalizando 30 muestras. En el momento de la recolección, con el auxilio de termómetro infrarrojo fue realizada la medida de la temperatura de exposición, fueron encaminas para el Núcleo de Ciencia y Tecnología de Alimentos del Instituto de Investigaciones Científicas y Tecnológicas del Estado de Amapá-IEPA. Los análisis microbiológicos realizados fueron la presencia / ausencia de Salmonella spp. en $25 \mathrm{~g}$ de muestra y el recuento estándar de Staphylococcus coagulasa positiva (UFC / g). En cuanto a la temperatura de exposición fue posible constatar que 8 $(88,89 \%)$ de las muestras evaluadas estaban con temperaturas arriba de $4^{\circ} \mathrm{C}$, no atendiendo a la legislación vigente. En cuanto a los análisis microbiológicos realizados en este estudio se constató ausencia en todas las muestras para Salmonella spp., pero resultados positivos en el $100 \%$ de los establecimientos evaluados para Staphylococcus coagulasa positiva, donde la amplitud registrada fue de $2,3 \times 10^{3}$ a $2 \times 10^{5}$ UFC / g. Los supermercados incluso utilizando procesos mecanizados durante las etapas productivas y pasando por un patrón de control considerado más exigente que otros establecimientos, aún fue posible contactar un número expresivo de contaminación por Staphylococcus, que, aunque la legislación no prevea este microorganismo, considerado uno de los agentes patógenos más involucrados con brotes y casos esporádicos de intoxicaciones alimentarias.

Palabras clave: Salmonella, Staphylococcus, carne procesada

\section{Introdução}

A carne bovina é um alimento essencial para a dieta humana, sendo uma importante fonte de proteína animal (Almeida et al., 2015). Dentre as suas formas de apresentação, a carne moída possui excelente aceitação dos consumidores por 
diversos aspectos, tais como praticidade e preços acessíveis (Mendonça \& Silva, 2012). Segundo o Anexo II da Instrução Normativa $\mathrm{N}^{\mathrm{o}} 83$, de 21 de novembro de 2003 este produto cárneo é definido como resultante da moagem de massas musculares de carcaças bovinas, com posterior resfriamento ou congelamento, sendo a sua venda permitida se a moagem for feita na presença do consumidor (Brasil, 2003a). Todavia, em muitos estabelecimentos comerciais é possível encontrar a carne pré-moída, que já permanece moída e pronta para a venda (Rosina \& Monego, 2013).

Apesar de ser um produto altamente nutritivo, é um alimento de rápida deterioração, por possuir grande quantidade de nutrientes, elevada atividade de água, maior área de contato exposta e $\mathrm{pH}$ favorável para a proliferação de microrganismos (Hangui et al., 2015). Além disso, como é obtido de pedaços de várias carnes, necessita de grande manipulação, o que o deixa exposto por um longo período em temperatura ambiente, aumentando o risco de contaminação (Nascimento et al., 2014). Para Velho et al. (2015), a deterioração neste produto é na maior parte das vezes imperceptível, dificultando que o consumidor perceba que a carne está inadequada.

O IBGE (2016) afirma que foram abatidos 29,7 milhões de bovinos em todo o país no ano de 2016, liderando o número de abates os estados de Mato Grosso, Mato Grosso do Sul, Goiás, São Paulo, Minas Gerais, Pará, Rondônia e Rio Grande do Sul, com cerca de $76,6 \%$ dos abates do país. A produção total de carnes em 2016/17 está estimada em 28,5 milhões de toneladas e a projeção para o final da próxima década é produzir 34,3 milhões de toneladas (Brasil, 2017). Essas projeções para o Brasil mostram que esse setor deve apresentar intenso crescimento nos próximos anos com previsão de $2,1 \%$ ao ano na produção de carnes bovinas.

No Brasil, a RDC N ${ }^{\circ} 12$ de 2 de janeiro de 2001 (Brasil, 2001) preconiza apenas a ausência de Salmonella spp. em $25 \mathrm{~g}$ de carne bovina in natura. Silva et al. (2016) ressaltam que embora não existam padrões estabelecidos para Staphylococcus coagulase positiva, a detecção destes microrganismos reforça a avaliação das condições sanitárias.

No Amapá, após inúmeros achados (SilvaJúnior et al., 2015a; Silva-Júnior et al., 2015b; Santos et al., 2016; Silva-Júnior et al., 2017a; Silva-Júnior et al., 2017b; Costa et al., 2018; Nascimento et al., 2018), a segurança de alimentos tem se tornado alvo de interesse pela comunidade científica. Por estes motivos, o objetivo deste trabalho foi analisar as características microbiológicas de carne bovina moída comercializada em supermercados da cidade de Macapá, Amapá.

\section{Material e Métodos}

Foram realizadas três coletas de amostras de carne bovina moída, as quais foram adquiridas em dez supermercados localizados em diferentes pontos da cidade de Macapá (Figura 1) durante os meses de fevereiro a julho de 2018, totalizando 30 amostras. O tamanho amostral foi calculado com o Software EpiInfo 7.2.2.6, considerando frequência esperada de $50 \%$, erro $\beta$ de $20 \%$ e erro $\alpha=0,01$.

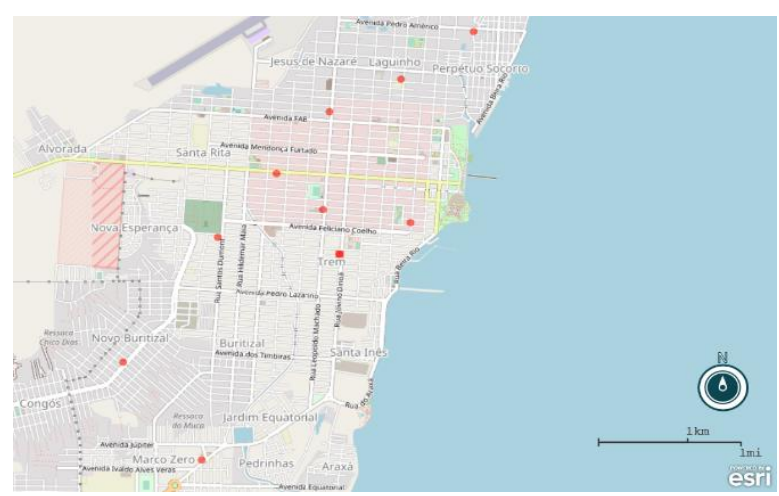

Figura 1. Localização dos supermercados selecionados para a coleta de amostras na cidade de Macapá, Amapá.

Cada amostra continha cerca de $500 \mathrm{~g}$ de carne e foram escolhidas aleatoriamente de balcões refrigerados dos supermercados. No momento da coleta, com o auxílio de termômetro infravermelho FLUKE 62MAX+ foi realizada a medida da temperatura de exposição. As amostras foram acondicionadas em caixa isotérmica, contendo gelo seco e encaminhadas para os Laboratórios de Microbiologia e Físico-Química do Núcleo de Ciência e Tecnologia de Alimentos do Instituto de Pesquisas Científicas e Tecnológicas do Estado do Amapá.

\section{Análises microbiológicas}

Os métodos analíticos foram baseados na Instrução Normativa $N^{\circ} 62$ (Brasil, 2003b), que oficializa os métodos de controle de produtos de origem animal e os resultados encontrados foram comparados com os padrões estabelecidos na RDC $n^{\circ}$ 12/2001 (Brasil, 2001).

De cada amostra analisada, procedeu-se a coleta asséptica de $25 \mathrm{~g}$ de carne moída que foram 
homogeneizadas em $225 \mathrm{~mL}$ de água peptonada a 0,1\% estéril (HIMEDIA, RM001), com auxílio de homogeneizador (Stomacher). De cada amostra foi realizada a diluição decimal seriada.

Para a análise de Staphylococcus coagulase positiva (SCP) foram utilizadas as três diluições de cada amostra, das quais $0,1 \mathrm{~mL}$ foi inoculado na superfície de placas contendo Ágar BairdParker (BP) com o auxílio de uma alça de Drigalski. As placas foram incubadas a $35-37^{\circ} \mathrm{C}$ por 45 a 48 horas. Após este período, foram contadas as colônias típicas para os testes de catalase, coagulase e coloração de Gram. Foi calculado o número UFC/g em função do número de colônias típica contadas, diluição inoculada e percentual de colônias confirmadas.

Para a determinação de ausência/presença de Salmonella spp. foram pesados $25 \mathrm{~g}$ de cada amostra e foi transferida para saco estéril de homogeneização e acrescentado $225 \mathrm{~mL}$ de água peptonada tamponada. Após homogeneização, as amostras foram incubadas a $37 \pm 1^{\circ} \mathrm{C}$ por $18 \pm 2$ horas. Após incubação, alíquotas de $0,1 \mathrm{~mL}$ e 1 $\mathrm{mL}$ foram transferidas para os caldos Rappaport Vassiliadis Soja (incubado a $37 \pm 1^{\circ} \mathrm{C} / 24 \pm 3 \mathrm{~h}$ ) e caldo Tetrationato Muller Kauffmann Novobiocina (incubado a 41,5 $\pm 1^{\circ} \mathrm{C} / 24 \pm 3 \mathrm{~h}$ ) respectivamente. Dos tubos, foram alçadas por estria de esgotamento na superfície de ágar de Xilose Lisina Desoxicolato (ágar XLD) incubado a $37 \pm 1^{\circ} \mathrm{C} / 24 \pm 3 \mathrm{~h}$. A confirmação foi realizada com a transferência para ágar Três Açúcares e Ferro (TSI) com picada em profundidade e realizado movimentos de estrias na superfície, sendo incubado a $35 \pm 0,5^{\circ} \mathrm{C} / 18-24$ horas. Após este período foi passado por série bioquímica (Teste de fermentação da glicose, sacarose e lactose; teste indol; malonato; citrato; urease e descarboxilação da lisina em caldo). Das amostras que apresentaram características bioquímicas compatíveis com o gênero Salmonella spp. e que não apresentou auto-aglutinação, foi submetida à sorologia polivalente.

\section{Análises físico-químicas}

As análises físico químicas foram baseadas no recomendado pelo Instituto Adolfo Lutz (2008). O método potenciométrico foi utilizado para a determinação do pH. Foi pesado $50 \mathrm{~g}$ de cada amostra e homogeneizado em $150 \mathrm{~mL}$ de água destilada a $25^{\circ} \mathrm{C}$ e foi deixado em repouso durante 10 minutos. O sobrenadante foi transferido para um béquer e foi realizada a leitura do $\mathrm{pH}$ em um potenciômetro digital de bancada marca Tecnopon, modelo mPA-210. O pH de 5,8 a 6,2 foram considerados carnes boas para o consumo.

\section{Resultados e Discussão}

Na avaliação da temperatura da carne moída comercializada em supermercados da cidade de Macapá, Amapá foi possível constatar que 8 $(88,9 \%)$ das amostras avaliadas estavam fora da recomendação da legislação vigente, onde a Instrução Normativa $n^{\circ} 83$, de 21 de novembro de 2003 do MAPA (Brasil, 2003) estabelece que a refrigeração para carne moída deve ser mantida entre $0^{\circ} \mathrm{C} \mathrm{a} 4^{\circ} \mathrm{C}$. Estes dados estão próximos do padrão encontrado por outros autores. Lino et al. (2009) relataram 99,2\% dos pontos comerciais avaliados com vendas expostas fora da temperatura estabelecida em Jabotão dos Guararapes - Pernambuco. Arçari, et al. (2011) observaram que em $80 \%$ dos supermercados na cidade de Vitória - Espírito Santo estavam fora da legislação. Baptista et al. (2013) destacaram que apenas $10 \%$ das amostras de carne moída avaliadas na região metropolitana de Recife, Pernambuco apresentavam temperatura interna inferior à $4^{\circ} \mathrm{C}$. No trabalho realizado por Bonacina et al. (2017) em supermercados de Erechin - Rio Grande do Sul, $100 \%$ estavam com temperaturas acima de $4^{\circ} \mathrm{C}$. A temperatura de armazenamento da carne não pode ser ignorada, uma vez que se trata de um parâmetro essencial para a conservação do alimento, influenciando diretamente na velocidade da proliferação de microrganismo (Costa et al., 2012).

Em relação ao pH, $100 \%$ das amostras avaliadas (variação entre 5,8 a 6,0) estavam de acordo com o preconizado pelo MAPA (Brasil, 2003), onde determina que a carne bovina moída deve ter pH menor que 6,2. Souza et al. (2000), em amostras oriundas de açougues da cidade de Macapá - Amapá mencionaram 100\% em conformidade com a legislação. Velho et al. (2015) em amostras provenientes da cidade de Mossoró - Rio Grande do Norte encontraram valores que variaram entre 5,5 a 5,7. Por outro lado, no trabalho realizado por Oliveira et al. (2017), 85,0\% das amostras de carne moída bovina comercializada em açougues de Bom Jesus - Piauí estavam em desacordo com o pH, estando fora do limite entre 5,8 a 6,2. É importante destacar que o pH é um relevante parâmetro de qualidade, pois reflete diretamente na proliferação microbiana e na retenção de água (Oliveira et al., 
2017). Segundo Alcantara, Morais \& Souza (2012), pH baixo em carnes vai favorecer a multiplicação de bactérias láticas. O músculo no post-morten vai acidificar de acordo com o aumento da glicólise.

Quanto as análises microbiológicas realizadas neste estudo constataram-se resultados positivos em $100 \%$ dos estabelecimentos avaliados, onde a amplitude registrada foi de $2,3 \times 10^{3}$ a $2 \times 10^{5} \mathrm{UFC} / \mathrm{g}$ para Staphylococcus Coagulase positiva (Tabela 1). No trabalho realizado por Rosina \& Monego (2013), 95,0\% das amostras de carne moída provenientes de supermercados de Canoinhas, Santa Catarina foram positivas para S. Coagulase positiva; porém, $87,5 \%$ apresentaram contagens superiores a $10^{3} \mathrm{UFC} / \mathrm{g}$. Silvestre et al. (2013) detectaram Staphylococcus em todas as amostras de carne moída in natura avaliados em Alexandria, Rio Grande do Norte. Por outro lado, Gomes et al. (2017) detectaram em Montes Claros, Minas Gerais apenas uma amostra com valores elevados para $S$. Coagulase positiva. Acosta et al. (2017) destaca que contagens inferiores a $10^{3} \mathrm{UFC} / \mathrm{g}$ indicam condições inapropriadas de higiene e/ou processamento inadequado, contagens variando entre $10^{3}$ a $10^{4} \mathrm{UCF} / \mathrm{g}$ indicam risco a saúde, e contagens próximas a $10^{5} \mathrm{UFC} / \mathrm{g}$ gera risco epidemiológico, pois é compatível com o início da produção da enterotoxina. São conhecidos 20 tipos diferentes de enterotoxinas, mas sabe-se que sua produção é influenciada por fatores como temperatura e pH (Luz et al., 2015). Soares et al. (2015) afirma que a enterotoxina é termoestável, e mesmo que a carne moída passe por tratamento térmico antes do consumo, o mínimo para inativar a toxina deve ser de $100^{\circ} \mathrm{C}$ por 30 minutos. No presente estudo, as amostras $\mathrm{C}, \mathrm{G}$ e $\mathrm{H}$ apresentaram contagens superiores a $10^{5} \mathrm{UFC} / \mathrm{g}$.

Tabela 1. Resultado das análises físico-químicas e microbiológicas em carne moída comercializadas em supermercados.

\begin{tabular}{lcccc} 
Supermercado & $\mathrm{Ph}$ & $\mathbf{T}^{\mathbf{0}}$ & $\begin{array}{c}\mathrm{SCP} \\
(\mathrm{UFC} / \mathrm{g})\end{array}$ & $\begin{array}{c}\text { Salmonella } \\
\text { sp. }\end{array}$ \\
\hline $\mathrm{A}$ & $5,82 \pm 0,13$ & $8,1^{\circ} \mathrm{C}$ & $5,33 \times 10^{3}$ & Ausente \\
$\mathrm{B}$ & $5,91 \pm 0,03$ & $13,5^{\circ} \mathrm{C}$ & $1 \times 10^{4}$ & Ausente \\
$\mathrm{C}$ & $6,03 \pm 0,04$ & $5^{\circ} \mathrm{C}$ & $1,5 \times 10^{5}$ & Presente \\
$\mathrm{D}$ & $5,87 \pm 0,25$ & $2^{\circ} \mathrm{C}$ & $2,3 \times 10^{3}$ & Ausente \\
$\mathrm{E}$ & $5,85 \pm 0,04$ & $6^{\circ} \mathrm{C}$ & $3 \times 10^{3}$ & Ausente \\
$\mathrm{F}$ & $5,94 \pm 0,02$ & $6^{\circ} \mathrm{C}$ & $5,5 \times 10^{4}$ & Ausente \\
$\mathrm{G}$ & $5,91 \pm 0,5$ & $10^{\circ} \mathrm{C}$ & $2 \times 10^{5}$ & Ausente \\
$\mathrm{H}$ & $5,86 \pm 0,08$ & $9^{\circ} \mathrm{C}$ & $1,1 \times 10^{5}$ & Ausente \\
I & $5,86 \pm 0,16$ & $7,1^{\circ} \mathrm{C}$ & $1,9 \times 10^{4}$ & Ausente \\
Padrão* & $<6,2$ & $0-4^{\circ} \mathrm{C}$ & - & Ausente \\
\hline & & & &
\end{tabular}

Para Salmonella spp. Todas as amostras foram ausentes, assim como recomendado pela legislação (Brasil, 2001). Esse resultado foi semelhante ao observado por Luz et al. (2015) em carnes moídas comercializadas em Natal, Rio Grande do Norte. Em estudo realizado por Fritzen et al. (2006), em carnes moídas comercializadas no estado do Paraná, 69,6\% estavam fora do padrão estabelecido.

Os supermercados mesmo utilizando processos mecanizados durante as etapas produtivas e passando por um padrão de controle considerado mais exigente do que outros estabelecimentos, ainda foi possível contatar um número expressivo de contaminação por Staphylococcus, que mesmo que a legislação não preveja este microrganismo, ele ainda é considerado um dos agentes patogênicos mais envolvidos com surtos e casos esporádicos de intoxicações alimentares.

\section{Conclusão}

Em 100\% das amostras foi constatada a presença de Staphylococcus coagulase positiva, e em três destas amostras apresentaram contagens superiores a $10^{5} \mathrm{UFC} / \mathrm{g}$, sendo compatível com o início da produção da toxina estafilocócica. As altas contagens podem ser justificadas pela higienização inadequada de equipamentos e utensílios.

Mesmo que a contagem de $S$. coagulase positiva não esteja prevista na $\operatorname{RDC} \mathrm{n}^{\circ} 12$ da Agência Nacional de Vigilância Sanitária para carne bovina moída, é perceptível que este produto pode se tornar um importante veiculador de intoxicações alimentares estafilocócicas, sendo necessário um rígido controle higiênico-sanitário em todas as etapas de seu processamento, associado à educação sanitária dos manipuladores e consumidores.

\section{Referências}

Acosta, A. C., Costa, M. M., Pinheiro-Júnior, J. W. \& Mota, R. A. 2017. Fatores de virulência de Staphylococcus aureus. Medicina Veterinária (UFRPE), 11(4), 252-269.

Alcantara, M., Morais, I. C. L. \& Souza, C. M. O. C. C. 2012. Principais microrganismos envolvidos na deterioração das características sensoriais de derivados cárneos. Revista Brasileira de Higiene e Sanidade animal, 6(1), 1-18.

Almeida, B. S., Monteiro, W. A. \& Bezerra, F. Y. P. 2015. Perfil Microbiológico da carne moída comercializada no Município de Juazeiro do 
Norte, Ceará. Revista Interfaces: Saúde, Humanas e Tecnologia, 3(1), 1-8.

American Public Health Association - APHA. 2001. Committee on Microbiological for Foods. Compendium of methods for the microbiological examination of foods. 4th ed. Washington: Alpha.

Arçari, A.T., Junior, G. M. \& Beltrame, M.A.V. 2011. Avaliação microbiológica da carne bovina moída comercializada em cinco supermercados de Vitória, ES. Higiene Alimentar, 25(202/203), 138-144.

Baptista, R. I. A. A., Moura, F. M. L., Fernandes, M. F. T. S., Santos, V. V. M \& Fernandes, E. F. T. S. 2013. Aspectos qualitativos da carne moída comercializada na região metropolitana do Recife-PE. Acta Veterinária Brasílica, 7(1), 38-47.

Bonacina, M.S., Baccin, M. A. \& Rosa, L. S. 2017. Avaliação de parâmetros indicativos da qualidade da carne bovina moída comercializada em diferentes supermercados em Erechin, Rio Grande do Sul. Revista Vigilância Sanitária em debate, 5(4), 9-16.

Brasil, 2001. Ministério da Saúde, Agência Nacional de Vigilância Sanitária (ANVISA). Resolução - RDC $n^{\circ} 12$, de 2 de janeiro de 2001. Regulamento técnico sobre padrões microbiológicos para alimentos. Diário Oficial da União. Brasília, DF.

Brasil, 2017. Ministério da Agricultura, Pecuária e Abastecimento. Projeções do Agronegócio Brasil 2016/17 a 2026/27 projeções de longo prazo. 8. Ed. Brasília - DF, p.125.

Brasil. 2003. Ministério da Agricultura, Pecuária e Abastecimento. Instrução Normativa $n^{\circ} 83$, de 21 de novembro de 2003. Aprova os regulamentos técnicos de identidade e qualidade de carne bovina em conserva e carne moída de bovino. Diário Oficial da União. Brasília, DF.

Costa, A.L.P., Nascimento, J.F. \& Silva-Júnior, A.C.S. Perfil de resistência de Staphylococcus aureus isolados de pescada amarela (Cynoscion acoupa) comercializada em feira pública. PubVet. 12(5), a84, 1-6.

Costa, J.N.P., Santos, V.V.M., Silva, G.R., Moura, F.M.L., Siqueira, M.G.F.M., Gurgel, C.A.B. \& Moura, A.P.B.L. 2012. Condições de armazenamento e acondicionamento de carnes in natura comercializadas em minimercados. Medicina Veterinária, 6(4), 10-15.

Fritzen, A.L., Schwerz, D.L., Gabiatti, E.C., Padilha, V. \& Macari, S.M. 2006. Análise microbiológica de carne moída de açougues pertencentes a $9^{\circ}$ Regional de Saúde do Paraná. Higiene Alimentar, 20,144.

Gomes, A.F.A., Almeida, E.E.S., Souza, S.A., Silva, J.P., Amâncio, T.A., Santos, C.C., Barbosa, R.P., Oliveira, F.S. \& Farias, P.K.S. 2017. Avaliação microbiológica de carnes moídas bovinas em diferentes estabelecimentos comerciais. Caderno de Ciências Agrárias, 9(3), 95-100.

Hangui, S.A.R., Ferreira, A.F., Dourado, A.T.S., Martins, J.D., Vargem, D.S. \& Silva, J.R. 2015. Análise microbiológica da carne bovina moída comercializada na cidade de Anápolis, Goiás, Brasil. Revista Eletrônica de Farmácia, 12(2), 30-38.

IBGE. 2018. Levantamento sistemático da produção agrícola (LSPA). Disponível em: http://www.ibge.gov.br/home/estatistica/indic adores/agropecuaria/lspa/. Acessado em: 25 fev. 2018

Instituto Adolfo Lutz (São Paulo). 2008. Métodos físico-químicos para análise de alimentos/coordenadores Odair Zenebon, Neus Sadocco Pascuet e Paulo Tiglea -- São Paulo: Instituto Adolfo Lutz, p. 1020.

Lino, G.C., Pacheco, M.S., Rolim, M.B.Q., Paiva, J.N. \& Moura, A.P.B.L. 2009. Condições higiênico-sanitárias dos estabelecimentos de comercialização de carnes nos mercados públicos de Jaboatão dos Guararapes, PE. Medicina Veterinária. 3(4), 1-6.

Lutz, N. A. d. I. A. (2005). Métodos químicos e físicos para análise de alimentos (Vol. 1): 4th ed. São Paulo, Brazil.

Luz, J.R.D., Araújo, J.H.L., Batista, D., Silva, T.C., Araújo, L.B.A. \& Carvalho, C.T. 2015. Qualidade microbiológica da carne moída comercializada em Natal, Rio Grande do Norte. Nutrivisa - Revista de Nutrição e Vigilância em Saúde, 2(2), 86-90.

Mendonça B. S.\& Silva C. S. 2012. Qualidade microbiológica da carne moída comercializada na cidade Cariacica, ES. Higiene Alimentar, 26(208/209), 101-105. 
Nascimento, J.F., Barroso, B.S., Tostes, E.M.L., Silva, A.S.S. \& Silva-Júnior, A.C.S. 2018. Análise físico-química de polpas de acerola (Malpighia glabra L.) artesanais e industriais congeladas. PubVet. 12(6, a109), 1-6.

Nascimento, M.V.D., Uedes, A.T.L., Silva, H.A., Santos, V.E.P. \& Paz, M.C.F. 2014. Avaliação microbiológica da carne moída fresca comercializada no mercado central em Campina Grande-PB. Revista Saúde e Ciência. 3(1), 56-68.

Oliveira, M.S., Souza, V.C., Pinto, C.O., Nunes, G.S., Natylane, E.F., Machado, F.C.F. \& Machado-Júnior, A.A.N. 2017. Qualidade físico-química e microbiológica da carne moída de bovino em açougues. Revista Electrónica de Veterinaria. 18(12), 1-13.

Rosina, A. \& Monego, F. 2013. Avaliação microbiológica da carne bovina moída nas redes de supermercados de Canoinhas/SC. Revista Saúde e Meio Ambiente, 2(2), 55-64.

Santos, R.G., Dias, M.C.D., Porcy, C. \& Galeno, N.S. 2016. Identificação de fungos produtores de micotoxinas cancerígenas em pães de sanduíches vendidos no centro comercial de Macapá-AP. Revista da Associação Brasileira de Nutrição. 7(2), 50-55.

Silva, J.B., Prazeres, A.R., Oliveira, A.C.S., Dantas, V.V., Barros, M.C.S., Silva, F., Abel, I., Roos, T. B. \& Moraes, C. M. 2016. Avaliação higiênico-sanitária de estabelecimentos comerciais e análise de micro-organismos indicadores em amostras de carne bovina (coxão mole) in natura comercializadas em mercados públicos. Revista do Instituto Adolfo Lutz. São Paulo, 75(1709), 1-7.

Silva-Júnior, A.C.S., Barbosa, F.H.F., ProiettiJúnior, A.A., Palha, S.E.M. \& Emin, E.T. 2015b. Avaliação microbiológica de pescada branca (Cynoscion spp.) comercializada na feira do pescado, Macapá-AP. Higiene Alimentar, 29 (246/247).

Silva-Júnior, A.C.S., Malcher, E.S.T., Silva, A.S.S., Nascimento, J.F. \& Barroso, B.S. 2017a. Perfil de resistência a antimicrobianos de Staphylococcus aureus isolados de queijo manteiga comercializado em feiras públicas da cidade de Macapá, AP. Higiene Alimentar, 31, 274/275.

Silva-Júnior, A.C.S., Silva, A.S.S., Brito, T.P. \& Ferreira, L.R. 2015a. Ocorrência de Staphylococcus coagulase positiva e coliformes termotolerantes em Jaraqui, Semaprochilodus brama (Valenciennes, 1850) comercializado na Feira do Pescado, MacapáAP. Biota Amazônia, 5(1), 32-36.

Silva-Júnior, A.C.S., Silva, A.S.S., Soares, N.R.M., Moraes, G.R., Sousa, C.M. \& Nascimento, J.F. 2017b. Caracterização físicoquímica e avaliação microbiológica de concentrado proteico de peixe (Piracuí) comercializado em feiras livres da Cidade de Macapá-AP. Biota Amazônia. 7(3), 33-36.

Silvestre, M. K., Abrantes, M.R., Paiva, W.S., Souza, E.S. \& Silva, J.B.A. 2013. Avaliação da qualidade da carne bovina in natura comercializada no município de AlexandriaRN. Acta Veterinária Brasílica, 7(4), 327-331.

Soares, P. M. K., Silva, J.B.A., Souza, L.B., Mendes, C.G., Abrantes, M.R., Campelo, M.C.S. \& Souza, A.S. 2015. Qualidade microbiológica de carne bovina comercializada na forma de bife. Revista Brasileira de Ciência Veterinária, 22(3-4), 206-210.

Souza, C.L., Peixoto, M.R.S., Silva, E.C. \& Oliveira, R.I.S.R. 2000. Avaliação da qualidade microbiológica e físico-química da carne bovina em açougues do município de Macapá, AP. Higiene Alimentar, 14(72), 6065.

Velho, A. L. M. C. S., Abrantes, M. R., Medeiros, J. M. S., Aguiar, K. C. S., Sousa, E. S., Soares, K. M. P. \& Silva, J. B. A. 2015. Avaliação qualitativa da carne bovina in natura comercializada em Mossoró-RN. Acta Veterinária Brasílica, 9(3), 212-217.

Recebido: 3 Agosto, 2018.

Aprovado: 30 Agosto, 2018

Publicado: 22 Outubro, 2018

Licenciamento: Este artigo é publicado na modalidade Acesso Aberto sob a licença Creative Commons Atribuição 4.0 (CC-BY 4.0), a qual permite uso irrestrito, distribuição, reprodução em qualquer meio, desde que o autor e a fonte sejam devidamente creditados. 